\title{
La salud mental en los tiempos del coronavirus.
}

\author{
Mental health in the time of coronavirus.
}

\author{
Silvana Sarabia ${ }^{1, a ; 2, b ; c}$
}

Nunca pensé vivir una experiencia como la desencadenada por esta pandemia. Nunca imaginé no poder abrazar a mi papá en su $88^{\circ}$ cumpleaños (22/03), o no poder asistir a la graduación de Maestría de mi hija mayor (28/05) ya que ha sido cancelada. Estoy segura que todos tienen sus propios ejemplos de lo que no han podido ni podrán hacer debido al coronavirus. Cuarentena, distanciamiento social, aislamiento social, inmovilización obligatoria, aplanar la curva se han vuelto palabras del día a día. El coronavirus (COVID-19) ha cambiado nuestro estilo de vida y lo seguirá cambiando. Debido a esto surgen dudas e incertidumbres: ¿Cuándo podré regresar a mi rutina?, ¿Cuándo podré trabajar?, ¿Hasta cuándo se extenderá la cuarentena? No tenemos, ni tendremos, en corto tiempo, una respuesta definitiva, por lo que debemos ser resilientes, cuidar de nuestra salud mental, la de nuestra familia, la de nuestros pacientes $y$, en lo posible, la de la comunidad entera.

En los últimos meses se han publicado varios artículos sobre cómo cuidarnos y cómo cuidar la salud mental durante esta pandemia. Cuidarnos a nosotros mismos e incentivar el auto-cuidado en nuestros pacientes es parte de nuestra responsabilidad como psiquiatras. Una Guía (1) publicada para psiquiatras pero también aplicable a otros profesionales de la salud mental, recomienda: 1. Mantenerse al tanto de todo lo relacionado a la pandemia a fin de proporcionar información correcta a los pacientes (en nuestro medio se puede revisar la página web https://www. gob.pe/coronavirus); 2. Corregir la información errada: si los pacientes refieren datos incorrectos relacionados a la pandemia, corregir la idea equivocada y orientarlos correctamente; 3. Limitar la exposición a los medios de comunicación (y la redes sociales): se ha descrito que una exposición excesiva a los medios de comunicación puede generar consecuencias negativas en la salud mental, por lo que se sugiere recomendar a los pacientes (y profesionales de la salud) limitar tales contactos y tenerlos con medios confiables; 4. Educar a los pacientes, a sus familiares y a los profesionales de la salud sobre las respuestas psicológicas y conductuales relacionadas con los brotes infecciosos y las intervenciones para su manejo; 5. Proveer instrucción sobre las reacciones de estrés: a) Reconocer y normalizar las reacciones de estrés; b) Enseñar a los pacientes a reconocer sus propias reacciones y su manejo temprano; c) Discutir estrategias para reducir el estrés, tales como: i. Estar preparados y aplicar las medidas preventivas; ii. Tener un sueño reparador, alimentarse regularmente y realizar ejercicios; iii. Evitar el uso de alcohol, cigarrillos y drogas; iv. Conversar con familiares y amigos sobre sus preocupaciones; v. Practicar métodos de relajación; vi. Participar de actividades recreativas en casa; 6. Identificar a los pacientes de alto riesgo y asegurar que todos los pacientes tengan medicinas suficientes a fin de no interrumpir su tratamiento, y 7. Cuidarnos y cuidar a nuestra familia: atender las necesidades básicas, i.e. mantenerse hidratado, comer y dormir regularmente; tener periodos de descanso y practicar estrategias para reducir la ansiedad $(2,3)$. Debemos estar y sentirnos bien para poder ayudar eficazmente a otros.

La cuarentena terminará, la inmovilización obligatoria se levantará ya que ambas no son sostenibles a largo plazo por el daño social y económico que pueden ocasionarle al país. Pasarán muchos meses para retornar a lo

1 Revista de Neuro-Psiquiatría, Facultad de Medicina Alberto Hurtado, Universidad Peruana Cayetano Heredia. Lima, Perú.

2 Sección Académica de Psiquiatría y Salud Mental, Departamento Académico de Clínicas Médicas, Facultad de Medicina Alberto Hurtado, Universidad Peruana Cayetano Heredia. Lima, Perú.

a Editora Jefa ; ${ }^{b}$ Profesora ; ${ }^{\mathrm{C}}$ Médica-Psiquiatra. 
"normal" ya que, muy probablemente, el aislamiento social, el lavado de manos y otras medidas continuarán. Cuidemos nuestra salud mental, la salud mental de nuestros seres queridos, la de los profesionales de la salud y la de la comunidad (4). Estos son momentos en que, como profesionales de la salud, debemos cuidar a otros, pero es también nuestra obligación cuidar nuestra salud mental tanto como la física.

\section{REFERENCIAS BIBLIOGRÁFICAS}

1. Center for the Study of Traumatic Stress. Taking care of patients during coronavirus outbreak: A Guide for Psychiatrists. Bethesda, USA: Center for the Study of Traumatic Stress; 2020. (Citado el 22 de marzo de 2020) Disponible en: https://www. cstsonline.org/assets/media/documents/CSTS FS_Taking_Care_of_Patients_During_ Coronavirus_Outbreak_A_Guide_for Psychiatrists_03_03_2020.pdf

2. World Health Organization. Mental health and psychological considerations during the COVID-19 outbreak. Geneva: World Health Organization ; 2020. (Citado 23 de marzo del 2020) Disponible en: https://apps.who.int/iris/bitstream/handle/106 65/331490/WHO-2019-nCoV-MentalHealth2020.1-eng.pdf

3. Center for the Study of Traumatic Stress. Sustaining the well-being of healthcare personnel during coronavirus and other infectious disease outbreaks. Bethesda, USA: Center for the Study of Traumatic Stress; 2020. (Citado el 22 de marzo del 2020) Disponible en: https://www.cstsonline. org/assets/media/documents/CSTS_FS_ Sustaining_Well_Being_Healthcare_Personnel_
during.pdf.pdf

4. Xiang YT, Yang Y, Li W, Zhang L, Zhang Q, Cheung $\mathrm{T}, \mathrm{Ng} \mathrm{CH}$. Timely mental health care for the 2019 novel coronavirus outbreak is currently needed. Lancet Psychiatry. 2020;7(3):228-229.

\section{Páginas web de interés}

Center for the Study of Traumatic Stress:

https://www.cstsonline.org/resources/resourcemaster-list/coronavirus-and-emerging-infectiousdisease-outbreaks-response

Mental health and psychological considerations during the COVID-19 outbreak:

https://apps.who.int/iris/bitstream/ handle/ $10665 / 331490 /$ WHO-2019-nCoV-

MentalHealth-2020.1-eng.pdf

Guía de intervención humanitaria mhGAP (GIHmhGAP. El manejo clínico de los trastornos neurológicos y por uso de sustancias en las emergencias humanitarias:

https://iris.paho.org/handle/10665.2/28418 